\title{
Drenagem linfática no pós operatório de lipoaspiração de abdome: uma revisão da literatura
}

A busca por tratamentos estéticos é constante na população brasileira e a lipoaspiração de abdome é a mais realizada. Para a melhora dos resultados a cirurgia é indicada a Drenagem Linfática Manual (DLM) no pós-operatório, que tem o objetivo de prevenir possíveis sequelas e promover uma melhor recuperação. 0 principal objetivo é revisar na literatura sobre a eficácia da drenagem linfática manual no pós operatório de lipoaspiração de abdome. Consultando artigos científicos publicados nas bases indexadas: U.S. National Library of Medicine National Institutes Health (Pubmed) e Scientific Electronic Library Online (SciELO) e com a ferramenta de busca Google Scholar, sem restrição de data. Através dos artigos e livros analisados neste estudo, foi possível comprovar a eficácia da DLM no pós-operatório de lipoaspiração. Conclui-se com esta revisão que, a DLM é um método eficaz para a melhora do quadro de sintomas como seromas, fibrose e lindedemas, causados pelo impacto do processo cirúrgico. Além da melhora dos sintomas, a técnica é capaz de estimular o sistema imunológico e acelerar o processo de cicatrização das incisões.

Palavras-chave: Drenagem linfática; Pós Operatório; Lipoaspiração de Abdome; Cirurgia.

\section{Lymphatic drainage in the post-surgery of abdominal liposuction: a revision of the literature}

\begin{abstract}
The search for aesthetic treatments is constant in the Brazilian population and abdominal liposuction is the most performed. For the improvement of the results the surgery is indicated the Manual Lymphatic Drainage (DLM) in the postoperative, that has the objective to prevent possible sequels and to promote a better recovery. The main objective is to review the literature on the efficacy of manual lymphatic drainage in the postoperative period of abdominal liposuction. Consultation of scientific articles published in the databases indexed: U.S. National Library of Medicine National Institutes Health (Pubmed) and Scientific Electronic Library Online (SciELO) and with the search tool Google Scholar, without date restriction. Through the articles and books analyzed in this study, it was possible to prove the efficacy of DLM in the postoperative period of liposuction. It is concluded with this review that, DLM is an effective method for the improvement of symptoms such as seromas, fibrosis and lindedemas, caused by the impact of the surgical process. In addition to improving the symptoms, the technique is able to stimulate the immune system and accelerate the healing process of the incisions.
\end{abstract}

Keywords: Lymphatic drainage; Post Operative; Abdomen Liposuction; Surgery.

Topic: Enfermagem Geral

Reviewed anonymously in the process of blind peer.
Received: $12 / 12 / 2017$

Approved: 05/02/2018

Cleuma Regina Freitas de Almeida Pontes

Instituto de Excelência em Educação e Saúde, Brasil.

cleuma.regina@hotmail.com

Luiz Aurélio Rodrigues Watanabe

Instituto de Excelência em Educação e Saúde, Brasil.

http://lattes.cnpq.br/6877886751156526

cleuma.regina@hotmail.com

Referencing this:

PONTES, C. R. F. A.; WATANABE, L. A. R.. Drenagem linfática no pós operatório de lipoaspiração de abdome: uma revisão da literatura. Scire Salutis, v.8, n.1, p.37-43, 2018. DOI: http://doi.org/10.6008/CBPC2236-9600.2018.001.0005 


\section{INTRODUÇÃO}

Procedimentos cirúrgicos são realizados rotineiramente nos hospitais brasileiros, sejam procedimentos cirúrgicos de emergência, eletivos ou estéticos, então, por ser um procedimento agressivo, o sistema linfático sofre alterações que causam o linfedema, uma das complicações em procedimentos cirúrgicos, já que o trajeto da linfa é alterado. Assim a Drenagem Linfática Manual (DLM) é a técnica utilizada para a melhora no quadro de linfedemas, assim como outras complicações. A DLM é o método terapêutico amplamente utilizado para drenar o excesso de líquido do interstício celular, visando restaurar a capacidade de transporte do sistema linfático e seu equilíbrio. Trata-se de uma massagem leve, superficial, com manobras especificas seguindo o trajeto do sistema linfático (ALENCAR et al., 2009).

Vodder e Leduc são as duas técnicas representadas na DLM, e em ambas são realizadas as seguintes manobras: captação, reabsorção e evacuação da linfa. Todas essas manobras são realizadas de forma lenta, com movimentos suaves, relaxantes de intermitentes (SCHWUCHOW et al., 2008). Alencar et al. (2009) afirmam que a drenagem linfática manual deve ser aplicada a todos os pacientes que sejam submetidos a processos cirúrgicos, sejam eles estéticos ou não. A aplicação da técnica objetiva o reestabelecimento do sistema linfático, que é alterado de forma agressiva durante procedimentos cirúrgicos. A técnica promove além da melhora do edema, prevenção de complicações como fibroses, seromas, linfedemas, necrose, cicatrizes hipertróficas ou queloideana, hematomas e sofrimento cutâneo. Além destas prevenção citadas, o paciente submetido a drenagem linfática manual bem executada é capaz de promover diversos resultados, como a melhora na recuperação pós-cirúrgica e prevenção de doenças do sistema imune.

Com a preocupação constante com relação a estética corporal, muitas pessoas buscam a cirurgia plástica para reparar aqui que incomoda na aparência e a lipoaspiração de abdome é o procedimento mais procurado pelos brasileiros que buscam o corpo perfeito. Sua aplicação promove a melhora no contorno abdominal sem que seja realizada a ressecção da pele e pode ser utilizada em outras partes do corpo, mas quando realizado esse procedimento, ocorre o trauma da área por onde as cânulas passam, provocando sangramento e a formação do edema. Por ser um procedimento subcutâneo, os vasos linfáticos são amplamente afetados e com isso ocorrem a formação do linfedema (MARTINS et al., 2007).

A drenagem linfática manual tornou-se uma técnica amplamente utilizada e indicada por vários profissionais por oferecer ao paciente qualidade de vida e prevenção de possíveis sequelas pós cirúrgicas. Diante disso, este estudo tem como objetivo revisar na literatura sobre a eficácia da drenagem linfática manual no pós operatório de lipoaspiração de abdome.

\section{METODOLOGIA}

Esta revisão bibliográfica foi realizada através da consulta a artigos científicos publicados nas bases indexadas: U.S. National Library of Medicine National Institutes Health (Pubmed) e Scientific Electronic Library Online (SciELO) e com a ferramenta de busca Google Scholar, sem restrição de datas. Os descritores 
utilizados foram: 'Drenagem linfática', 'Pós operatório', 'Lipoaspiração de abdome' e 'Cirurgia' em português e em inglês.

\section{DISCUSSÃO TEÓRICA}

Intervenções cirúrgicas são realizadas para tratar doenças, lesões ou deformidades, e após esta intervenção o sistema linfático tende a funcionar de forma anormal, pois os ductos linfáticos são agredidos devido a ruptura dos tecidos (ZANELLA et al., 2011).

Cirurgia provém do latim chirurgia, que o tomou do grego kheirourgia, de kheír, mão + érgon, trabalho. Etimologicamente, portanto, cirurgia significa trabalho manual, arte, ofício, no qual se empregam as mãos para a sua execução. Entende-se, assim, o seu uso em medicina para designar os procedimentos terapêuticos que exigem trabalho manual.

Cirurgias podem ser realizadas por duas razões: médicas ou estéticas. Ambas são realizadas com o intuito de corrigir alterações e imperfeições, mas quando o paciente é submetido a intervenções cirúrgicas, ocorrem alterações significativas no funcionamento do sistema linfático comprometendo ou alterando-o dando início a formação do edema (ALENCAR et al., 2009). Assim, a técnica de lipoaspiração foi criada nos anos 80 , um procedimento realizado com pequenas incisões com o objetivo de drenar gordura localizada através de cânulas que geram uma pressão negativa, pois, com esse método, é possível retirar gordura em qualquer lugar do corpo, mas é importante ressaltar que a/o paciente deverá ter boa elasticidade da pele. Além do fator anteriormente citado, a Sociedade Brasileira de Cirurgia Plástica propõe um limite para a retirada de gordura, que consiste em $7 \%$ do peso corporal do paciente. Por se tratar de um procedimento subcutâneo, os vasos linfáticos são amplamente agredidos, e em consequência disso a formação de linfedemas.

O sistema linfático faz parte do sistema circulatório, sendo responsável por drenar um determinado tipo de fluido, a linfa. Trata-se de uma rede complexa de órgãos formados por: capilares linfáticos, vasos linfáticos, troncos linfáticos, ductos linfáticos e linfangion. Essa rede de vasos é responsável por encaminhar as células nutrientes e remover impurezas (GODOY et al., 1999; GODOY et al., 2006).

Os linfonodos são responsáveis por reter partículas estranhas, pois são responsáveis pela iniciação das defesas imunológicas. Além da função imunológica, o sistema linfático é uma reserva funcional do sistema venoso, com isso, quando o sistema venoso é incapaz de drenar líquidos, esta função passa a ser do sistema linfático, porem quando a quantidade de liquido ultrapassa a capacidade de drenagem do sistema linfático, ocorre a formação do edema/ linfedema (GODOY et al., 2006).

De acordo com Carvalho et al. (2006), o linfedema caracteriza-se pelo acumulo excessivo/anormal de líquidos e proteínas nos tecidos, decorrente da falha do sistema linfático, podendo estar associado a outras complicações. Em determinadas localidades do corpo, como membros superiores e inferiores, se não tratado o linfedema o mesmo poderá evoluir para o estágio de elefantíase. Edemas linfáticos, retenção hídrica, fibroedema gelóide e edemas pós-operatórios, são alterações do sistema linfáticos candidatos a aplicação da drenagem linfática manual e o profissional deve estar atento as essas alterações para a melhora da qualidade de vida do paciente. 
A drenagem linfática manual (DLM) é uma técnica de massagem responsável por retirar excesso de liquido através da mobilização da linfa, aonde regiões do corpo são afetadas por edemas. A drenagem é capaz de melhorar a circulação dos tecidos, aumenta a absorção de hematomas, acelera a cicatrização de ferimentos, além de melhorar o retorno da sensibilidade que é perdida depois de procedimentos cirúrgicos. (SCHWUCHOW et al., 2008).

As técnicas mais conhecidas da DLM são a de Vodder e Leduc, que podem ser realizadas de forma individual ou conjunta. A técnica Vodder foi desenvolvida por Emil Vodder e sua esposa Astrid, na década de 30, mas, inicialmente, a técnica era realizada em pacientes que adquiriam infecções das vias aéreas superiores aonde apresentavam gânglios cervicais edemaciados e duros, porém, ao verem os efeitos positivos nos pacientes, a técnica tornou-se bastante popular e com isso os Vodder viram-se na posição de aperfeiçoar a técnica para melhores resultados. A técnica utilizada por Vodder consiste numa combinação de movimentos passivos, de forma suave, lenta e repetitiva aonde segue o trajeto do fluxo linfático (BORGES, 2006; ALENCAR et al., 2009).

A técnica proposta por Leduc consiste na combinação de movimentos utilizando manobras básicas que direcionam a linfa para os coletores linfáticos e linfonodos, mas a captação ou reabsorção e manobras de evacuação ou demanda, são as manobras indicadas por Leduc que ainda propõe a aplicação da DLM de acordo com o tipo de distúrbio encontrado e ainda combina a técnica com a utilização de bandagens compressivas para melhores resultados (BARROS, 2001).

Além de possuir um conjunto de manobras complexas, a técnica deve obedecer a uma pressão especifica, de 30 a $40 \mathrm{mmHg}$, além de respeitar o trajeto correto do sistema linfático, prevenindo um possível colapso do mesmo. A execução correta da drenagem pode trazer benefícios que vão de estéticos como anticelulite, anti-estresse, pré e pós-parto, antienvelhecimento; terapêuticos para a melhora de resultados no pós operatório, tratamento de cicatrizes recentes, má circulação, retenção hídrica, dismenorreia e linfedema. A técnica é contra indicada para pessoas que sofrem de trombose venosa profunda, idade avançada, doenças malignas, pessoas que estejam sofrendo de algum processo inflamatório, neurites, erupções de pele, circulação descompensada e infecções ou ferimentos que estejam vigentes (ALENCAR et al., 2009).

Alencar et al. (2009) realizaram um estudo com o objetivo de analisar a utilização da drenagem linfática manual no pós-operatório de pacientes que se submeteram a cirurgia vascular de membros inferiores. A frequência de atendimentos foi de seis vezes por semana com média de tempo de quarenta e cinco minutos e a técnica de escolha foi a de LEDUC, que combina cinco movimentos formando seu sistema de massagem. Foi analisado através de uma escala a quantidade de dor sentida durante as sessões, e em todas a paciente não se referiu a dor nem durante ou término do procedimento. De acordo com os autores, a critério médico, a drenagem só foi realizada 72 horas após o procedimento cirúrgico, de modo suave devido a sensibilidade da região e evitar o aumento de lesões. Após sete sessões pode-se observar melhora na recuperação do aspecto saudável e normal da pele, diminuição de hematomas, reparação das incisões e favorecimento no processo de cicatrização. Para os autores é necessário que o profissional saiba o manejo correto da técnica para sua realização, além de conhecer a anatomia, fisiologia do sistema linfático e a 
histologia. Os autores afirmam que a drenagem linfática manual deve ser aplicada a todos os pacientes que sejam submetidos a processos cirúrgicos, sejam eles estéticos ou não. A aplicação da técnica objetiva o reestabelecimento do sistema linfático, que é alterado de forma agressiva durante procedimentos cirúrgicos. A técnica promove além da melhora do edema, previne complicações como fibroses, seromas, linfedemas, necrose, cicatrizes hipertróficas ou queloideana, hematomas e sofrimento cutâneo. Além da prevenção das complicações anteriormente citadas, o paciente submetido a drenagem linfática manual bem executada é capaz de promover diversos resultados, como a melhora na recuperação pós-cirúrgica e prevenção de doenças do sistema imune.

Soares et al. (2005) comparou os resultados da drenagem linfática manual (DLM) em relação a drenagem linfática mecânica (DLME) em pacientes submetidas a abdominoplastia- dermolipectomia. Foram realizadas sessões com tempo mínimo de 40 minutos, acompanhadas com exame físico e anamnese, assim iniciadas no 80 dia com término no 260 dia de pós-cirúrgico, por indicação médica. Através do procedimento, o fisioterapeuta observou parâmetros como: hematoma, edema, parestesia, algia e aderência cicatricial, além de demonstrar a redução de edema, perimetria e a satisfação das clientes. Todas as pacientes que participaram do estudo apresentaram algum tipo de alteração clínica no pós-operatório como: dor, equimoses, parestesia, edema e seroma.

Foi observado que após duas sessões de drenagem, seja ela manual ou mecânica, redução dos sintomas e melhora após dez atendimentos para a maioria das pacientes. Duas alterações de pós-operatorio persistiram, mesmo que de forma moderada, após as dez sessões: edema e parestesia. Soares et al. (2005) confirma que esse número de sessões não são o suficiente para eliminar todos os sintomas de pós-operatório. Foi constatado no estudo que a drenagem linfática manual obteve mais resultados na redução de edema, produzindo eliminação satisfatória de liquido. $\mathrm{O}$ autor conclui que as duas técnicas foram eficientes, porém a DLM obteve melhores resultados nos sintomas, perimetria e satisfação do cliente.

Schwuchow et al. (2008) incluíram no seu estudo, mulheres com idades entre 20 e 50 anos, que foram submetidas a lipoaspiração de tronco, logo todas as pacientes foram submetidas a 11 sessões de DLM, aonde na primeira avaliação todas elas apresentavam dor na região operada, edema, equimoses e fibrose, aspectos comuns de pós-operatório. Por ser uma técnica suave, nenhuma das pacientes relatou dor durante o procedimento, todas relataram a melhora no quadro de augias. Schwuchow et al. (2008), a DLM é indicada por médicos no pós operatório para prevenir a formação de fibroses e redução de edemas. Os resultados encontrados pelos autores demonstraram diminuição de medidas após todas as sessões de drenagem, tornando a DLM um método eficaz e preventivo de complicações da lipoaspiração.

Diante dos casos encontrados na literatura que foram anteriormente citados, Arieiro et al. (2007) afirmam que ao realizar cirurgias nos casos de câncer de cabeça e pescoço ocorrem algumas complicações como o linfedema, ocasionando ao paciente desconforto e sofrimento, além de provocar danos na fala, deglutição e dificuldades para a cicatrização do local cirurgiado. Para buscar métodos para aliviar os sintomas, os autores realizaram um estudo para verificar a eficácia da drenagem linfática manual no pós-operatório de câncer de cabeça e pescoço em três pacientes internados na enfermaria do Instituto Brasileiro de Controle 
do Câncer (IBCC). Durante o estudo, os autores realizaram a drenagem e optaram pelas vias occipto-axilares, pois eram as mais próximas das regiões acometidas pelo câncer. Nos dias de internação na enfermaria, os pesquisadores observaram grande diminuição do linfedema com a aplicação da drenagem linfática manual, e foi incluído diuréticos como método auxiliar. Porém os autores afirmam que é necessário um maior número de sessões para melhorar a qualidade de vida do paciente no período de pós operatório.

A drenagem linfática manual pode ser associada a outros métodos terapêuticos como a radiofrequência e o estudo de Bianchetti et al. (2015) teve como objetivo verificar a eficácia da terapia combinada de radiofrequência e a drenagem linfática, em uma paciente, que apresentava percentual de gordura localizada e hipotonia tissular abdominal. Na primeira avaliação, o adipômetro foi utilizado para avaliar as dobras cutâneas, perimetria com a utilização da fita métrica, utilização de bioimpedância para observação da composição corporal, peso e altura, registro fotográfico para avaliação posterior, teste de flacidez e diástase abdominal. Após as 10 sessões realizadas, os autores observaram melhora no aspecto do abdômen e redução da distensão abdominal. Para Bianchetti et al. (2015), a associação das técnicas junto com a realização de exercícios físicos potencializam a redução da gordura localizada, e com o auxílio nutricional os resultados podem ser ainda mais satisfatórios.

Silvestre et al. (2009) analisaram a eficácia da associação da drenagem linfática manual com o ultrassom para o tratamento de fibro edema gelóide, mais conhecido como celulite, em seis pacientes do sexo feminino numa clínica escola de fisioterapia da cidade de Concordia - SC. Sabe-se que esta patologia acarreta ao paciente uma visão estética desagradável, além de causar diminuição da funcionalidade e algías nas regiões afetadas. Para Silvestre et al. (2009), a associação das técnicas traz benefícios como a diminuição dos danos causados pela celulite e por ser um procedimento indolor, não invasivo e sem efeitos colaterais, eles ainda observaram, com o estudo, ótimos resultados com a associação das técnicas, já que ambas se complementam, pois o ultrassom ativa a circulação e o metabolismo linfático, e a drenagem linfática manual é capaz de eliminar toxinas e renovar tecidos.

No pós operatório da lipoaspiração de abdome, a associação das técnicas citadas podem potencializar os efeitos benéficos trazendo melhores resultados. Os casos clínicos apresentados no estudo faz compreender que a DLM é uma técnica eficaz no processo de recuperação pós cirúrgica, pois auxilia e beneficia a recuperação de forma mais rápida, podendo observar que em muitos casos, a quantidade mínima de sessões (10 sessões) pode não ser eficaz para a melhora do quadro clínico de linfedema, sendo assim indicado mais sessões.

\section{CONCLUSÕES}

Pode-se concluir com este estudo que a DLM é um método efetivo para a recuperação pós-cirúrgica de lipoaspiração de abdome, bem como de outras cirurgias, pois, a técnica, é capaz de promover uma recuperação mais rápida e obter melhores resultados, já que tem ação de prevenir principalmente a formação de fibroses, equimoses, edema e acelerar o processo de cicatrização. A aplicação da técnica é 
segura, mas o profissional deverá estar amplamente capacitado para sua melhor execução e assim obter os melhores resultados.

\section{REFERÊNCIAS}

ALENCAR, T. P.; MEJA, D. P. M.. A influência da drenagem linfática manual no pós-operatório imediato de cirurgia vascular de membros inferiores. Monografia (Pós-graduação em Fisioterapia Dermato Funcional) - Faculdade Ávila, 2009.

ARIEIRO, E. G.; MACHADO, K. S.; LIMA, V. P.; TACANI, R. E.; DIZ, A. M.. A eficácia da drenagem linfática manual no pósoperatório de câncer de cabeça e pescoço. Rev. Bras. Cir. Cabeça Pescoço, v.36, n.1, p. 43-46, 2007.

BARROS, M. H.. Fisioterapia: Drenagem Linfática Manual. São Paulo: Robe, 2001.

BIANCHETTI, P. B.; SULZBACH, C.; KOLLET, F.; BLAU, M.; TASSINARY, J. A. F.; SINIGAGLIA, G.. Utilização de radiofrequência, terapia combinada, drenagem linfática manual e plataforma vibratória em hipotonia tissular e adiposidade localizada: estudo de caso. Caderno pedagógico, Lajeado, v.12, n.3, p.140-149, 2015.

BORGES, F. S.. Dermato-funcional: Modalidades terapêuticas nas disfunções estéticas. São Paulo: Phorte, 2006.

CARVALHO, T. F.; BATISTA, H. S.; GODOY, J. M. P.. Linfedema e mastectomia: Vamos conversar sobre isso?. Rio de Janeiro: Di Livros, 2006.

GODOY, M. F. G.; GODOY, J. M. P.; BRAILE, D. M.. Tratamento do linfedema de membros superiores: Atividades e Exercícios Linfomiocinéticos. Rio de Janeiro: Di Livros, 2006.
GODOY, J. M. P.; GODOY, M. F. G.. Drenagem linfática manual: uma nova técnica. São José do Rio Preto: Lin Comunicação, 1999.

MARTINS, E. A.; MIRANDA, L. F. D.; SAKAE, T. M.; VALLE, L. F. C.; ELY, J. B.. Avaliação de uma série de 38 casos de pacientes submetidos à cirurgia de lipoaspiração em Tubarão- SC entre outubro de 2004 e fevereiro de 2005. Arquivos Catarinenses de Medicina, v.36, n.1, 2007.

SCHWUCHOW, L.; SOUZA, V. P.; CALOY, L.; RESENDE, T. L.. Estudo do uso da drenagem linfática manual no pósoperatório da lipoaspiração de tronco em mulheres. Revista da Graduação Publicações de TCC, v.1, n.1, 2008.

SILVESTRE, C. P.; ZANON, C. S.. O uso do ultra-som associado com a drenagem linfática manual no tratamento do fibro edema gelóide. Ágora: R. Divulg. Cient., Mafra, v.16, n.2, 2009.

SOARES, L. M. A.; SOARES, S. M. B.; SOARES, A. K. A.. Estudo comparativo da eficácia da drenagem linfática manual e mecânica no pós-operatório de dermolipectomia. RBPS, v.18 n.4, p.199-204, 2005.

ZANELLA, B. I.; RUCKL, S.; VOLOSZIN, M.. A importância da drenagem linfática manual no pós-operatório da abdominoplastia. Monografia (Bacharelado em Tecnologia em Cosmetologia e Estética) - Universidade do Vale do Itajaí, 2011.

A CBPC - Companhia Brasileira de Produção Científica (CNPJ: 11.221.422/0001-03) detém os direitos materiais desta publicação. Os direitos referem-se à publicação do trabalho em qualquer parte do mundo, incluindo os direitos às renovações, expansões e disseminações da contribuição, bem como outros direitos subsidiários. Todos os trabalhos publicados eletronicamente poderão posteriormente ser publicados em coletâneas impressas sob coordenação da Sustenere Publishing, da Companhia Brasileira de Produção Científica e seus parceiros autorizados. Os (as) autores (as) preservam os direitos autorais, mas não têm permissão para a publicação da contribuição em outro meio, impresso ou digital, em português ou em tradução. 